\title{
Erratum to: The Role of Antipsychotics in the Management of Fibromyalgia
}

\author{
Elena P. Calandre • Fernando Rico-Villademoros
}

Published online: 17 May 2013

(c) Springer International Publishing Switzerland 2013

\section{Erratum to: CNS Drugs (2012) 26(2):135-153 DOI 10.2165/11597130-000000000-00000}

The authors have alerted us to the following errors:

Page 148, column 1, paragraph 2, lines 4-13: The following three sentences, which previously read:

There were no significant differences between quetiapine and amitriptyline treatments in terms of the mean changes from baseline in the scores of the FIQ $(p=0.7065)$, PSQI $(\mathrm{p}=0.5758)$ and BDI $(\mathrm{p}=0.9227)$. Although quetiapine was not inferior with respect to amitriptyline in terms of efficacy, there were a higher number of dropouts due to adverse events among quetiapine-treated patients (42\%) than among those who received amitriptyline (13\%).

should read:

There were no significant differences between quetiapine and amitriptyline treatments in terms of the mean changes from baseline in the scores of the FIQ $(\mathrm{p}=0.7065)$, PSQI $(\mathrm{p}=0.5758)$ and BDI $(\mathrm{p}=0.9227)$. However, quetiapine did not meet the criterion for noninferiority and there were a higher number of dropouts due to adverse events among quetiapine-treated patients (42\%) than among those who received amitriptyline (13\%).

Page 148, column 1, section 3.3, paragraph 1, lines 5-12: The following sentence, which previously read:

The online version of the original article can be found under doi:10.2165/11597130-000000000-00000.

E. P. Calandre $(\square) \cdot$ F. Rico-Villademoros

Instituto de Neurociencias y Centro de Investigaciones

Biomédicas, University of Granada, Avenida de Madrid 11,

18012 Granada, Spain

e-mail: calandre@gmail.com
Although we have to wait until full data from all four quetiapine trials are available, preliminary results from two randomized clinical trials $[137,138]$ seem to indicate that this drug provides a significant relief of the overall symptomatology in patients with fibromyalgia, and that this improvement is similar to that provided by amitriptyline.

should read:

Although we have to wait until full data from all four quetiapine trials are available, preliminary results from two randomized clinical trials $[137,138]$ seem to indicate that this drug provides a significant relief of the overall symptomatology in patients with fibromyalgia; however, in one of these trials the improvement with quetiapine did not meet the criterion to be considered noninferior as compared to amitriptyline.

Page 149, column 1, section 4, paragraph 2, lines 15-21: The following sentence, which previously read:

Preliminary results from two of the four randomized clinical trials performed using quetiapine for this indication suggest that this drug provides significant relief to the overall symptomatology in patients with fibromyalgia and that this improvement is similar to that obtained using amitriptyline.

should read:

Preliminary results from two of the four randomized clinical trials performed using quetiapine for this indication suggest that this drug provides significant relief to the overall symptomatology in patients with fibromyalgia, although in one trial this improvement was not noninferior to that obtained using amitriptyline. 